\title{
Association of serum adipocyte fatty acid-binding protein and apolipoprotein B /apolipoprotein A1 ratio with intima media thickness of common carotid artery in dyslipidemic patients
}

\author{
Jiri Orsag ${ }^{a}$, David Karasek ${ }^{a}$, Milan Halenka ${ }^{a}$, Helena Vaverkova ${ }^{a}$, Jaromira Spurna ${ }^{a}$, Veronika Kubickova ${ }^{b}$, Jiri Lukes ${ }^{b}$, \\ Josef Zadrazila
}

\begin{abstract}
Background. Diseases caused by atherosclerosis play the most important role in mortality and morbidity worldwide. Serum adipocyte fatty acid binding protein (A-FABP) seems to be a new promising marker to determine the risk of atherosclerosis.

Objective. The aim of this study was to evaluate relationships between serum A-FABP levels in studied individuals and to assess the possibility of modeling the intima media thickness of the common carotid artery (C-IMT) using A-FABP levels and other observed characteristics.

Methods. Seventy two Caucasian individuals were enrolled and divided into 3 groups: dyslipidemic patients with or without metabolic syndrome (MetS+, $n=17$; MetS-, $n=34$ ) and controls $(n=21)$.

Results. There was confirmed the well-established risk profile of individuals with MetS (unfavorable lipid and lipoprotein profile, as well as increased parameters of insulin resistence and C-IMT). A-FABP concentrations in this group were significantly higher in comparison with both MetS- and controls.

Conclusion. Using multiple linear regression models of C-IMT values for all individual data, healthy controls and dyslipidemic patients without metabolic syndrome (MetS-) A-FABP levels were not revealed as an important predictor of C-IMT in our model. In contrast, age, gender, waist circumference, nonHDL cholesterol levels and ApoB/ApoA1 ratio were important repressors of C- IMT in study individuals. This finding may be attributed to the overwhelming effect of other more robust risk factors for atherosclerosis in these individuals.
\end{abstract}

Key words: adipocyte fatty acid binding protein, intima media thickness, dyslipidemia

Received: April 8, 2018; Accepted: July 19, 2018; Available online: September 11, 2018

https://doi.org/10.5507/bp.2018.043

aDepartment of Internal Medicine III - Nephrology, Rheumatology and Endocrinology, Faculty of Medicine and Dentistry, Palacky University Olomouc and University Hospital Olomouc, Czech Republic

${ }^{b}$ Department of Clinical Biochemistry, University Hospital Olomouc, Czech Republic

Corresponding author: Jiri Orsag, e-mail:jiri.orsag@fnol.cz

\section{INTRODUCTION}

Diseases caused by atherosclerosis such as stroke or myocardial infarction play the most important role in mortality and morbidity worldwide, especially in highly developed countries. New markers for onset of atherosclerosis are sought. Serum adipocyte fatty acid binding protein (A-FABP) seems to be a new promising marker to determine the risk of atherosclerosis.

Adipocyte fatty acid-binding protein (A-FABP) is a member of the fatty acid binding protein super family, accounting for approximately $6 \%$ of total cellular proteins in mature adipocytes ${ }^{1}$. It is also present in macrophages, which possess similar functions to adipocytes, modulated by proliferator-activated receptor-c agonists and oxidized low density lipoproteins ${ }^{2}$. Recently, ectopic expression and function of A-FABP in several other types of cells and tissues (for example endothelial cells of capillaries and small veins in the heart and kidney) have been demonstrated $^{3}$. A-FABP-deficient mice are protected from insulin resistance, hyperglycemia, and atherosclerosis ${ }^{4,5}$. It has been demonstrated that A-FABP is released into the human blood stream ${ }^{6}$.

Human serum A-FABP levels are significantly higher in men than in women, possibly because of the larger amount of body fat in women ${ }^{6,7}$. Its circulating levels are elevated in patients with obesity and other components of the metabolic syndrome, including adverse lipid profiles, hyperglycemia and hypertension, independently of sex, age and adiposity ${ }^{6,8-10}$. Serum A-FABP levels correlate also positively with markers of endothelial dysfunction, and coronary atherosclerotic burden ${ }^{11-15}$.

High concentration of A-FABP at baseline was an independent predictor for the development of metabolic syndrome during a five-year follow-up period in a Chinese population ${ }^{16}$. A 10-year prospective study also showed that high level of A-FABP at baseline independently predicted the development of type 2 diabetes ${ }^{17}$. Associations of A-FABP levels with the development of left ventricular (LV) hypertrophy and with systolic and diastolic cardiac dysfunction have also been reported ${ }^{18-21}$. These findings support the role of A-FABP as a key pro-inflammatory 
mediator that links obesity with cardiovascular damage and atherosclerosis.

The aim of this cross sectional study was to evaluate relationships between serum A-FABP levels in studied individuals and to assess the possibility of modeling intima media thickness of common carotid artery (C-IMT) by A-FABP levels and other observed characteristics.

\section{METHODS}

\section{Study design and subjects}

The study cohort included asymptomatic dyslipidemic Czech subjects and healthy volunteers who underwent C-IMT measurement in the Lipid Centre of the Department of Internal Medicine III, University Hospital Olomouc, Czech Republic. The medical history was obtained and physical examination was performed, including measurement of waist circumference, body mass index (BMI), and systolic and diastolic pressure (SBP, DBP). Patients with the following conditions were excluded from the study: secondary hyperlipidemia (hypothyroidism, renal or hepatic diseases, nephrotic syndrome), hypolipidemic therapy in the previous 6 weeks, hormone therapy, acute infection or trauma and history of clinically manifested atherosclerosis (coronary artery disease, cerebrovascular disease or peripheral arterial disease). Individuals who met these criteria ( 72 subjects, 31 men and 41 women) were divided into three groups: 17 hyperlipidemic patients with the metabolic syndrome (MetS+, men/women: $8 / 9$, mean age: $47.9 \pm 14.5$ years $), 34$ hyperlipidemic patients without the metabolic syndrome (MetS-, men/women: 16/18, mean age: $40.8 \pm 15.5$ years) and 21 normolipidemic healthy subjects (men/women: $7 / 14$, mean age: $47.0 \pm 17.3$ years) who served as a control group. A diagnosis of metabolic syndrome was based on a modified version of the National Cholesterol Education Program Adult Treatment Panel (NCEP ATP III) and defined as at least three of the following at baseline: waist circumference $($ men $>102 \mathrm{~cm}$, women $>88 \mathrm{~cm})$, triglycerides $(\mathrm{TG}) \geq 1.7 \mathrm{mmol} / \mathrm{L}, \mathrm{HDL}$ cholesterol (men $<1.04 \mathrm{mmol} / \mathrm{L}$, women $<1.30 \mathrm{mmol} / \mathrm{L}$ ), blood pressure $\geq 130 / \geq 85 \mathrm{~mm} \mathrm{Hg}$ and fasting glucose $\geq 6.1 \mathrm{mmol} / \mathrm{L}$. Presence of metabolic syndrome was evaluated at the time of first patient examination in the lipid center. The study was reviewed and approved by the Ethics Committee of the Faculty of Medicine and Dentistry and University Hospital Olomouc. Informed consent was obtained from all participants.

\section{Laboratory analysis}

All subjects were assessed after overnight fasting for at least $12 \mathrm{~h}$. Venous blood samples were obtained and after centrifugation, the serum was used for analysis. Routine serum biochemical parameters were analyzed in the day of blood collection, concentrations of adipokines were measured in the sample aliquots stored at $-80{ }^{\circ} \mathrm{C}$, no longer than 6 months. Total cholesterol (TC), TG and high density lipoprotein cholesterol (HDL-C) were determined enzymatically on Modular SWA system
(Roche, Basel, Switzerland). HDL-C was measured by direct method without precipitation of apoB containing lipoproteins. Low density lipoprotein cholesterol (LDL-C) levels were calculated using Friedewald formula. NonHDL-cholesterol (nonHDL-C) was calculated as TC HDL-C. Concentration of apoB and apolipoprotein A1 (apoA1) were determined immunoturbidimetrically using Tina-Quant ApoB and ApoA-1 kits (Roche, Basel, Switzerland). Glucose was measured using GOD-PAP method (Roche, Basel, Switzerland). A-FABP was determined using Human A-FABP ELISA kit (Biovendor Laboratory Medicine Inc., Brno, Czech Republic).

\section{Measurement of carotid IMT}

High-resolution B-mode ultrasound (Philips Sonos 5500,2004 ) was used to measure the IMT of the common carotid arteries (CCA). Linear array transducers with frequency of $10 \mathrm{MHz}$ were used. Anterolateral approach was used to longitudinally image the right and left CCA. This image was displayed just before the widening of the bulb. When an optimal longitudinal image of the far wall of the CCA in the region of $1 \mathrm{~cm}$ proximally from the bulb was obtained, it was frozen on the $\mathrm{R}$ wave according to a simultaneous ECG and video tapered. Three video records were made on both CCA. C-IMT measurements were processed off-line using the software Image-Pro plus (Version 4.0, Media -Cybernetics, Silver Spring, USA). The region under evaluation was the CCA wall $1-2 \mathrm{~cm}$ distant proximally from the mentioned border. The average of all mean C-IMT of three frozen images of both sides was chosen as outcome variable. Plaque was defined as a focal protrusion into the lumen with a thickness of at least $50 \%$ more than adjacent intima-media complex. Subjects with this atherosclerotic plaque in the evaluated region were not included in the study. The measurement of C-IMT was made without knowledge of laboratory results.

\section{Statistical analysis}

Statistical analyses were performed with software R, ver. 3.4.2. (ref. $^{22}$ ). Data were summarized as means and standard deviation and analysed using ANOVA, Student's t-test, Kruskall-Wallis or Wilcoxon test. In the case of more than two groups, multiple comparisons were done with Tukey test, resp. Nemenyi test. Categorical variables were analyzed with Pearson's chi-square test, or Fisher's exact test. Multiple linear models of IMT values were built for all individuals' data and also for subset of healthy controls and dyslipidemic patients without metabolic syndrome (MetS-). Feature selection was based on LASSO (Least Absolute Shrinkage and Selection Operator) regression results for lambda parameter obtained as minimal value in 5-fold cross-validation process for each analyzed set of data ( $\mathrm{R}$ package glmnet) ( ref. $^{23}$ ). Linear model for dyslipidemic patients with metabolic syndrome was not performed due to empty non-zero coefficient set of Lasso regression results for these subset of patients. In suitable cases (existence of non-significant regressors), a stepwise selection process was also performed. In case of healthy subset, based on fit diagnostic plot (Cook's distance), one leverage observation was excluded in final model. 


\section{RESULTS}

The demographic, clinical and biochemical characteristic of the investigated groups are summarized in Table 1 and Fig. 1, 2. Individuals with MetS (MetS+) had highest $\mathrm{BMI}$ and waist circumference and expected unfavorable lipid and lipoprotein profiles (elevated TC, TG, and non-
HDL-C, ApoB, ApoB/ApoA1 ratio and decreased HDL-C and ApoA1) and signs of insulin resistance (increased levels of insulin and C-peptide). A-FABP concentrations in this group were significantly higher in comparison with both MetS- and controls. Differences in A-FABP levels between MetS- and controls were not significant. Similar results were found in C-IMT ( significantly thicker C-IMT

Table 1. Clinical and biochemical characteristics of study subjects.

\begin{tabular}{|c|c|c|c|}
\hline Parameters & $\begin{array}{c}\text { Controls } \\
n=21\end{array}$ & $\begin{array}{l}\text { MetS- } \\
n=34\end{array}$ & $\begin{array}{c}\text { MetS+ } \\
\mathrm{n}=17\end{array}$ \\
\hline Age (years) & $47.0 \pm 17.3$ & $40.8 \pm 15.5$ & $47.9 \pm 14.5$ \\
\hline A-FABP $(\mu \mathrm{g} / \mathrm{L})$ & $21.6 \pm 11.0^{c}$ & $21.5 \pm 9.5^{\mathrm{c}}$ & $38.4 \pm 30.1^{\mathrm{a}, \mathrm{b}}$ \\
\hline C-IMT (mm) & $0.60 \pm 0.12^{\mathrm{c}}$ & $0.60 \pm 0.14^{\mathrm{c}}$ & $0.80 \pm 0.13^{\mathrm{a}, \mathrm{b}}$ \\
\hline $\mathrm{SBP}(\mathrm{mm} \mathrm{Hg})$ & $129.4 \pm 14.3$ & $122.0 \pm 14.3^{\mathrm{c}}$ & $139.3 \pm 16.1^{b}$ \\
\hline $\mathrm{DBP}(\mathrm{mm} \mathrm{Hg})$ & $77.8 \pm 7.8$ & $75.6 \pm 9.5$ & $81.6 \pm 8.7$ \\
\hline $\operatorname{BMI}\left(\mathrm{kg} / \mathrm{m}^{2}\right)$ & $24.2 \pm 3.0^{\mathrm{c}}$ & $24.8 \pm 3.6^{\mathrm{c}}$ & $29.7 \pm 4.0^{\mathrm{a}, \mathrm{b}}$ \\
\hline Waist circumference $(\mathrm{cm})$ & $78.5 \pm 8.7^{\mathrm{c}}$ & $86.0 \pm 11.4^{\mathrm{c}}$ & $100.5 \pm 9.9^{\mathrm{a}, \mathrm{b}}$ \\
\hline hs-CRP (mg/L) & $1.8 \pm 2.4^{\mathrm{c}}$ & $2.9 \pm 3.5$ & $3.4 \pm 3.8^{\mathrm{a}}$ \\
\hline Total cholesterol (mmol/L) & $5.9 \pm 0.8^{\mathrm{b}, \mathrm{c}}$ & $7.4 \pm 1.4^{\mathrm{a}}$ & $7.2 \pm 1.9^{\mathrm{a}}$ \\
\hline Triglycerides (mmol/L) & $1.0 \pm 0.25^{\mathrm{b}, \mathrm{c}}$ & $2.3 \pm 1.75^{\mathrm{a}, \mathrm{c}}$ & $4.2 \pm 2.18^{\mathrm{a}, \mathrm{b}}$ \\
\hline LDL- cholesterol (mmol/L) & $3.6 \pm 0.76^{\mathrm{b}}$ & $4.9 \pm 1.32^{\mathrm{a}}$ & $4.3 \pm 1.6$ \\
\hline HDL-cholesterol (mmol/L) & $1.9 \pm 0.42^{\mathrm{b}, \mathrm{c}}$ & $1.5 \pm 0.44^{\mathrm{a}, \mathrm{c}}$ & $1.1 \pm 0.26^{\mathrm{a}, \mathrm{b}}$ \\
\hline nonHDL-cholesterol (mmol/L) & $4.0 \pm 0.75^{\mathrm{b}, \mathrm{c}}$ & $5.9 \pm 1.41^{\mathrm{a}}$ & $6.2 \pm 1.83 \underline{\mathrm{a}}$ \\
\hline $\operatorname{ApoA} 1(\mathrm{~g} / \mathrm{L})$ & $1.9 \pm 0.32^{\mathrm{c}}$ & $1.7 \pm 0.43$ & $1.4 \pm 0.26^{\mathrm{a}}$ \\
\hline $\operatorname{ApoB}(g / L)$ & $0.9 \pm 0.18^{\mathrm{b}, \mathrm{c}}$ & $1.4 \pm 0.29^{\mathrm{a}}$ & $1.4 \pm 0.38^{\mathrm{a}}$ \\
\hline ApoB/ApoA1 & $0.5 \pm 0.17^{\mathrm{b}, \mathrm{c}}$ & $0.9 \pm 0.30^{\mathrm{a}}$ & $1.0 \pm 0.26^{\mathrm{a}}$ \\
\hline Fasting glycaemia $(\mathrm{mmol} / \mathrm{L})$ & $4.9 \pm 0.37$ & $5.0 \pm 0.55$ & $5.5 \pm 0.91$ \\
\hline Insulin (mIU/L) & $7.4 \pm 4.86^{c}$ & $8.4 \pm 3.74^{\mathrm{c}}$ & $12.5 \pm 4.64^{\mathrm{a}, \mathrm{b}}$ \\
\hline C-peptide (mg/L) & $510.9 \pm 376.21^{\mathrm{c}}$ & $741.9 \pm 235.76^{\mathrm{c}}$ & $1026.4 \pm 345.66^{\mathrm{a}, \mathrm{b}}$ \\
\hline
\end{tabular}

MetS-, dyslipidemic patients without metabolic syndrome; MetS+, dyslipidemic patients with metabolic syndrome; A-FABP, adipocyte fatty acidbinding protein; IMT, carotid intima-media thickness; SBP, systolic blood pressure; DBP, diastolic blood pressure; BMI, body mass index; hs-CRP, high sensitivity C reactive protein; ApoA1, Apo lipoprotein A1; ApoB, Apo lipoprotein B

Data are presented as mean \pm standard deviation.

Differences in variables between subgroups were analyzed with ANOVA after adjustment for age and sex. Significant difference $P<0.05$ at least ${ }^{\mathrm{a}}$ vs. Controls; ${ }^{\mathrm{b}}$ vs. MetS-; ${ }^{\mathrm{v}}$ vs. MetS+

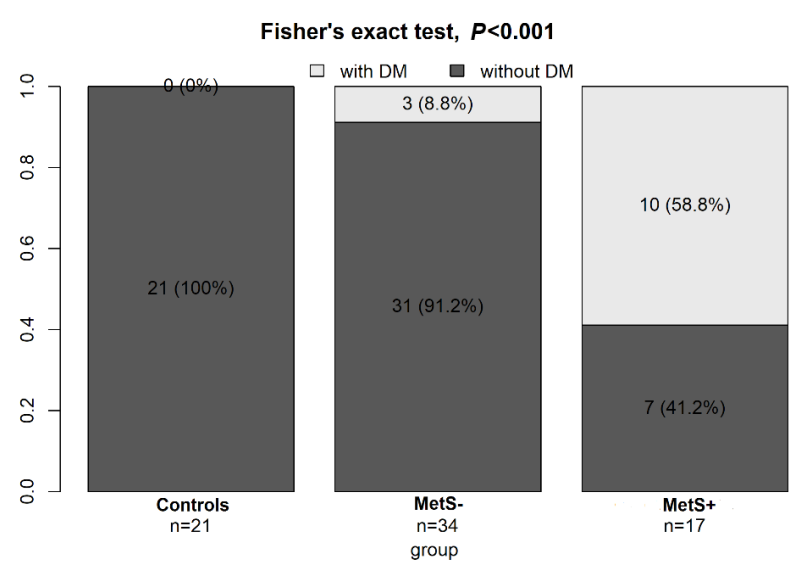

Fig. 1. Presence of diabetes mellitus in study groups.

MetS-, dyslipidemic patients without metabolic syndrome; MetS+, dyslipidemic patients with metabolic syndrome; DM, diabetes mellitus.
Pearson's test, $P=0.565$
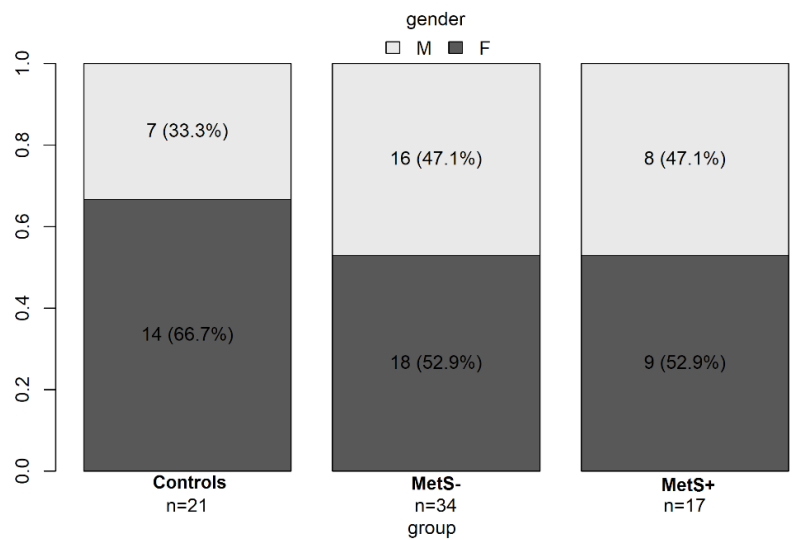

Fig. 2. Gender distribution of the studied groups.

MetS-, dyslipidemic patients without metabolic syndrome; MetS+, dyslipidemic patients with metabolic syndrome; M, male gender; F, female gender 
Table 2. Linear model of C-IMT mean for all study individuals $(n=65)$ after stepwise selection process for initial model with regressors: group, gender, hypertesion, age, waist circumference, nonHDL-cholesterol and ApoB/apoA1 (Multiple $\mathrm{R}^{2}=0.71$, Adjusted $\mathrm{R}^{2}=0.69$ ).

\begin{tabular}{lcccc}
\hline Coefficient & Estimate & Std. Error & \multicolumn{1}{c}{$P$} & Significance \\
\hline (Intercept) & 0.1148 & 0.0809 & 0.161 & NS \\
male gender & $\mathbf{0 . 0 5 1 3}$ & $\mathbf{0 . 0 2 5 4}$ & $\mathbf{0 . 0 4 8}$ & $\mathbf{0 . 0 5}$ \\
hypertesion & 0.0514 & 0.0275 & 0.0666 & NS \\
age & $\mathbf{0 . 0 0 5 0}$ & $\mathbf{0 . 0 0 0 7}$ & $\mathbf{0 . 0 0 0}$ & $\mathbf{0 . 0 0 1}$ \\
waist circumference & 0.0019 & 0.0011 & 0.0851 & NS \\
nonHDL-cholesterol & $\mathbf{0 . 0 2 5 8}$ & $\mathbf{0 . 0 7 1 0}$ & $\mathbf{0 . 0 0 0}$ & $\mathbf{0 . 0 0 1}$ \\
\hline Residuals: & & & & Max \\
Min & 1st quartile & Median & 3rd quartile & 0.2459 \\
-0.2480 & -0.0477 & 0.0014 & 0.0268 & \\
\hline
\end{tabular}

Table 3. Linear model of C-IMT mean for subset of controls $(n=18)$ after one leverage observation exclusion from initial model with the same regressors (Multiple $\mathrm{R}^{2}=0.89$, Adjusted $\mathrm{R}^{2}=0.87$ ).

\begin{tabular}{lcccc}
\hline Coefficient & Estimate & Std. Error & $P$ & Significance \\
\hline (Intercept) & 0.1434 & 0.0809 & 0.1610 & NS \\
waist circumference & $\mathbf{0 . 0 0 3 1}$ & $\mathbf{0 . 0 0 1 1}$ & $\mathbf{0 . 0 1 2 2}$ & $\mathbf{0 . 0 5}$ \\
\hline Residuals: & & & & \\
Min & 1st quartile & Median & 3rd quartile & Max \\
-0.0256 & -0.0255 & 0.0062 & 0.0162 & 0.0505 \\
\hline
\end{tabular}

Table 4. Linear model of C-IMT mean for subset of displipidemic patiens ( $\mathrm{n}=34)$ without metabolic syndrome MetS-

(Multiple $\mathrm{R}^{2}=0.72$, Adjusted $\mathrm{R}^{2}=0.7$ ).

\begin{tabular}{lcccc}
\hline Coefficient & Estimate & Std. Error & $P$ & Significance \\
\hline (Intercept) & $\mathbf{0 . 2 4 5 2}$ & $\mathbf{0 . 0 5 1 1}$ & $\mathbf{0 . 0 0 0 0}$ & $\mathbf{0 . 0 0 1}$ \\
age & $\mathbf{0 . 0 0 6 9}$ & $\mathbf{0 . 0 0 0 9}$ & $\mathbf{0 . 0 0 0 0}$ & $\mathbf{0 . 0 0 1}$ \\
ApoB/apoA1 & $\mathbf{0 . 1 3 8 4}$ & $\mathbf{0 . 0 4 5 6}$ & $\mathbf{0 . 0 0 4 8}$ & $\mathbf{0 . 0 1}$ \\
\hline Residuals: & & & & Max \\
Min & 1st quartile & Median & 3rd quartile & 0.1299 \\
\hline-0.1986 & -0.0359 & 0.0085 & 0.0384 & \\
\hline
\end{tabular}

in MetS+ than in MetS- and in controls, but no difference between MetS- and controls).

Using multiple linear regression models of C-IMT values for all individuals data and also for subset of healthy controls and dyslipidemic patients without metabolic syndrome (MetS-) A-FABP level was not revealed as an important predictor of C-IMT. Only age, gender, waist circumference, nonHDL cholesterol levels and ApoB/ ApoA1 ratio were important regressors of C-IMT see Table 2, 3, 4).

\section{DISCUSSION}

In the current study we confirmed the well-established risk profile of individuals with MetS. They had unfavorable lipid and lipoprotein profiles, as well as increased parameters of insulin resistence. A-FABP concentrations in this group were significantly higher in comparison with both MetS- and controls. This finding is consistent with the results of previous studies and our previous reports ${ }^{12}$.
It has been reported that increased circulating A-FABP levels are associated with obesity, insulin resistance, type 2 diabetes, hypertension, cardiac dysfunction, and atherosclerosis $^{6,14,16,17,24,25}$. High concentration of A-FABP at baseline was an independent predictor for the development of metabolic syndrome during a five-year follow-up period in a Chinese population ${ }^{16}$. A 10 -year prospective study also showed that high level of A-FABP independently predicted the development of type 2 diabetes ${ }^{17}$.

Relationship between serum A-FABP and C-IMT was observed in some previous studies. Hao Y. et al. revealed a positive correlation between serum A-FABP and subclinical atherosclerosis in a cohort of Chinese pre- and postmenopausal women with normal glucose tolerance ${ }^{26}$. A study of Yeung et al. reported that serum A-FABP levels were strongly associated with C-IMT in women including both hyperglycemia and normal glucose tolerance ${ }^{14}$. These findings support the notion that circulating A-FABP is not only a potent biomarker but also plays an important role, as an adipokine, in the development of atherosclerosis.

From this point of view is surprising, that A-FABP was 
not found as a predictor of C-IMT in our multiple linear regression models in contrast to classical factors as age, gender or waist circumference, even when all subjects were included in the analysis.

This is in contrast with study of Yeng et al. ${ }^{14}$, where multiple regression analysis had identified serum A-FABP together with age and hypertension to be independent risk factors related to C-IMT in female subjects. The study cohort consisted of many more subjects than ours (479 vs. 72 individuals). The other factor is separate testing of men and women. There was a difference in the race of participiants, too (Chinese vs.Caucasian). Other limitations of our study are as follows. First, we used a cross-sectional study design and therefore we could not establish a cause-and-effect relationship between A-FABP and C-IMT. Second, participants of our study were Caucasians, so our findings cannot be generalized to other ethnicities.

One other possible explanation for our findings is that the proatherosclerotic effects of conventional risk factors were stronger than A-FABP and probably outweighed its effect on carotid atherosclerosis. Therefore, future studies should be prospective and include a large number of cases.

One confounding factor in this study was the impossibility of using a linear model for dyslipidemic patients with metabolic syndrome due to empty non-zero coefficient set of Lasso regression results for this subset of patients. We only can speculate about the small number of individuals in MetS+ group and about the heterogeneity of these patients In any case, this phenomenon is surprising and we have no explanation for it.

An interesting fact is the $\mathrm{ApoB} / \mathrm{ApoA} 1$ ratio as an important predictor of C-IMT in dyslipidemic patients without metabolic syndrome.

The apoB/apoA1 ratio has been reported to be associated with the metabolic syndrome (MetS), and it may be a more convenient biomarker in MetS prediction, especially in Chinese populations ${ }^{27,28}$. In a single study on older healthy volunteers, Panayiotou et al. demonstrated that high ApoB/ApoA1 ratio is associated with early atherosclerosis $^{29}$. In linear regression analysis, the ApoB/ ApoA1 ratio was significantly associated with all the ultrasonic measurements of early atherosclerosis (among others C-IMT). In our study it is the first observation of the influence of ApoB/ApoA1 on C-IMT in dyslipidemic individuals.

\section{CONCLUSION}

Our data, in agreement with previous animal and clinical studies, revealed higher A-FABP levels and C- IMT in Czech dyslipidemic individuals with metabolic syndrome. This supports the role of A-FABP in the development of the metabolic syndrome and probably atherosclerosis. In contrast, to these findings A-FABP was not an important predictor of C-IMT in dyslipidemic patients. This finding may be attributed to the overwhelming effect of other risk factors of atherosclerosis in dyslipidemic individuals. One interesting finding is revealing of $\mathrm{ApoB} / \mathrm{ApoA} 1$ ratio as an important predictor of common C-IMT in dyslipidemic patients without metabolic syndrome.

Further investigations are needed to identify model regressors especially for very heterogenic groups of dyslipidemic patients with metabolic syndrome.

Acknowledgment: This work was supported by the IGA LF_2017_015 and MZ CR-RVO (FNOI, 00098892).

Authors contributions: JO, DK, MH, HV, JS took care of patients, reviewed the literature, drafted the manuscript, and contributed to its revision; VK, JL performed laboratory tests; JZ approved the manuscript.

Conflict of interest statement: None declared.

\section{REFERENCES}

1. Makowski L, Hotamisligil GS. Fatty acid binding proteins-the evolutionary crossroads of inflammatory and metabolic responses. J Nutr 2004; 134:2464-8.

2. Boord JB, Fazio S, Linton MF. Cytoplasmic fatty acid-binding proteins: emerging roles in metabolism and atherosclerosis. Curr Opin Lipidol 2002;13:141-7.

3. Elmasri H, Karaaslan C, Teper Y, Ghelfi E, Weng M, Ince TA, Kozakewich $\mathrm{H}$, Bischoff J, Cataltepe S: Fatty acid binding protein 4 is a target of VEGF and a regulator of cell proliferation in endothelial cells. FASEB J 2009;23:3865-73.

4. Boord JB, Maeda K, Makowski L, Babaev V, Fazio S, Linton MF Hotamisligil GS: Combined adipocyte-macrophage fatty acidbinding protein deficiency improves metabolism, atherosclerosis, and survival in apolipoprotein E-deficient mice. Circulation 2004; 110:1492-8.

5. Makowski L, Boord JB, Maeda K, Babaev VR, Uysal KT, Morgan MA, Parker RA, Suttles J, Fazio S, Hotamisligil GS, and Linton MF: Lack of macrophage fatty-acid-binding protein aP2 protects mice deficient in apolipoprotein E against atherosclerosis. Nat Med 2001;7:699-705.

6. Xu A, Wang Y, Xu JY, Stejskal D, Tam S, Zhang J, Wat NMS, Wong WK, Lam KSL: Adipocyte fatty acid-binding protein is a plasma biomarker closely associated with obesity and metabolic syndrome. Clin Chem 2006;52:405-13.

7. Ishimura S, Furuhashi M, Watanabe $Y$, Hoshina K, Fuseya T, Mita $T$, Okazaki Y, Koyama M, Tanaka M, Akasaka H, Ohnishi H, Yoshida H, and Saitoh S, Miura T: Circulating levels of fatty acid-binding protein family and metabolic phenotype in the general population. PLoS One 2013;8:e81318.

8. Stejskal D, Karpisek M. Adipocyte fatty acid binding protein in a Caucasian population: a new marker of metabolic syndrome? Eur J Clin Invest 2006;36:621-5.

9. Engl J, Ciardi C, Tatarczyk T, Kasr S, Laimer M, Laimer E, Weiss H, Aigner F, Molnar C, Tilg H, Patsch JR, Ebenbichler CF: A-FABP -a biomarker associated with the metabolic syndrome and/or an indicator of weight change? Obesity (Silver Spring) 2008;16:1838-42.

10. Hsu BG, Chen YC, Lee RP, Lee CC, Lee CJ, Wang JH. Fasting serum level of fatty -acid-binding protein 4 positively correlates with metabolic syndrome in patients with coronary artery disease. Circ J 2010;74:327-31.

11. Aragones G, Ferré R, Lázaro I, Cabré A, Plana N, Merino J, Heras $M$, and Girona J, Masana L: Fatty acid-binding protein 4 is associated with endothelial dysfunction in patients with type 2 diabetes. Atherosclerosis 2010;213:329-31.

12. Karasek D, Vaverkova H, Frysak Z, Orsag J, Novotny D, Halenka M, Slavik L: Relationship between serum adipocyte fatty acid-binding protein and endothelial/hemostatic markers in dyslipidemic subjects. Neuro Endocrinol Lett 2012;33(Suppl 2):26-31.

13. Miyoshi T, Onoue G, Hirohata A, Hirohata S, Usui S, Hina K, Kawamura H, Doi M, Kusano KF, Kusachi S, and Ninomiya Y: Serum adipocyte fatty acid-binding protein is independently associated with coronary atherosclerotic burden measured by intravascular ultrasound. Atherosclerosis 2010;11:164-9. 
14. Yeung DC, Xu A, Cheung CW, Wat NM, Yau MH, Fong CHY, Chau MT, Lam KSL: Serum Adipocyte Fatty Acid-Binding Protein Levels Were Independently Associated With Carotid atherosclerosis. Arterioscler Thromb Vasc Biol 2007;27:1796-1802.

15. Xiao $Y$, Yao L, and Li X, Zhong $H$, Chen XY, Tang WL, Liu SP, Xu AM, Zhou ZG: Relationship of adipocyte fatty acid-binding protein to adiponectin rations with femoral intima-media thickness and endothelium-dependent vasodilation in patients with newly-diagnosed type 2 diabetes mellitus. Zhonghua Yi ue Za Zhi 2010;90:231-5.

16. Xu A, Tso AW, Cheung BM, Wang Y, Wat NM, Fong CH, Yeung DC, Janus ED, Sham PC, and Lam KS: Circulating adipocyte-fatty acid binding protein levels predict the development of the metabolic syndrome: a 5-year prospective study. Circulation 2007;1 15:1537-43.

17. Tso AW, Xu A, Sham PC, Wat NM, Wang Y, Fong CH, Cheung BM, Janus ED, Lam KS: Serum adipocyte fatty acid binding protein as a new biomarker predicting the development of type 2 diabetes: a 10-year prospective study in a Chinese cohort. Diabetes Care 2007;30:266772.

18. Engeli S, Utz W, Haufe S, Lamounier-Zepter V, Pofahl M, Taber J, Janke J, Luft FC, Boschmann M, Schulz-Menger J, Jordan J: Fatty acid binding protein 4 predicts left ventricular mass and longitudinal function in overweight and obese women. Heart 2013;99:944-8.

19. Huang CL, Wu YW, Wu CC, Lin L, Wu YC, Hsu PY, Jong YS, Yang WS: Association between serum adipocyte fatty-acid binding protein concentrations, left ventricular function and myocardial perfusion abnormalities in patients with coronary artery disease. Cardiovasc Diabetol 2013;12:105.

20. Liu M, Zhou M, Bo Y, Xu Z, Li H, Zhang H, Zhu W, Zhang J, Xu A Wei $M$, Jia W: Circulating adipocyte fatty acid-binding protein levels are independently associated with heart failure. Clin Sci (Lond) 2013;124:115-22.

21. Baessler A, Lamounier-Zepter V, Fenk S, Strack C, Lahmann C, Loew T, Schmitz G, Bluher M, Bornstein SR, Fischer M: Adipocyte fatty acidbinding protein levels are associated with left ventricular diastolic dysfunction in morbidly obese subjects. Nutr Diabetes 2014;4:e106.
22. R Development Core Team : R: A language and environment for statistical computing. R Foundation for Statistical Computing, Vienna, Austria. ISBN 3-900051-07-0, 2008.

23. Tibshirani R, Bien J, Friedman J, Hastie T, Simon N, Taylor J, Tibshiran RJ: Strong Rules for Discarding Predictors in Lasso-type Problems. JRSSB 2012;74(2):245-66.

24. Ota H, Furuhashi M, Ishimura S, Koyama M, Okazaki Y, Mita T, Fuseya T, Yamashita T, Tanaka M, Yoshida H, Shimamoto K, Miura T: Elevation of fatty acid-binding protein 4 is predisposed by family history of hypertension and contributes to blood pressure elevation. Am J Hypertens 2012;25:1124-30.

25. Fuseya T, Furuhashi M, Yuda S, Muranaka A, Kawamukai M, Mita T, Ishimura S, Watanabe Y, Hoshina K, Tanaka M, Ohno K, Akasaka H, Ohnishi H, Yoshida H, Saitoh S, Shimamoto K, Miura T: Elevation of circulating fatty acid-binding protein 4 is independently associated with left ventricular diastolic dysfunction in a general population. Cardiovasc Diabetol 2014;13:126.

26. Hao Y, Ma X, Luo Y, Shen Y, Dou J, Pan X, Bao Y, Jia W: Serum Adipocyte Fatty Acid Binding Protein Levels Are Positively Associated With Subclinical Atherosclerosis in Chinese Pre- and Postmenopausal Women With Normal Glucose Tolerance. Clin Endocrinol Metab 2014;299:4321-7.

27. Zhong L, Li Q, Jiang Y, Cheng D, Liu Z, Wang B, Luo R, Cheng Q, Quing $\mathrm{H}$ : : The ApoB/ApoA1 ratio is associated with metabolic syndrome and its components in a Chinese population. Inflammation 2010;33:353-8.

28. Jing F, Mao Y, Guo J, Zhang Z, Li Y, Ye Z, Ding Y, Wang J, Jin M, Chen K: The value of Apolipoprotein B/Apolipoprotein $\mathrm{A} 1$ ratio for metabolic syndrome diagnosis in a Chinese population: a cross-sectional study. Lipids in Health and Disease 2014;13:81-7.

29. Panayiotou A, Griffin M, Georgiou N, Bond D, Tyllis T, TziakouriShiakalli C, Fessas C, Nicolaides A: ApoB/ApoA1 ratio and subclinical atherosclerosis. Int Angiol 2008;27(1):74-80. 